\title{
REMARKS ON ERGODIC THEOREMS
}

\author{
BY \\ EINAR HILLE
}

1. Introduction. In recent papers Nelson Dunford $[7,7 \mathrm{a}]\left({ }^{1}\right)$ has made a study of the spectral theory of linear bounded transformations on a Banach space to itself with a view to finding necessary and sufficient conditions for the convergence in various topologies of a sequence of linear transformations to a projection. This led him in particular to a systematic theory of ergodic theorems.

It is well known that in "discrete" ergodic theory one is concerned with the sequence of powers $T^{n}$ of a given transformation $T$. Ordinarily this sequence does not converge to a limit in the topology in question, but the sequence may be summable to a generalized limit. Classical ergodic theorems involve the use of $(C, 1)$ means, but arbitrary methods of summation have been considered by L. W. Cohen [6] and others.

The assertion that the sequence $\left\{T^{n}\right\}$ is summable by the method of summation $(S)$ is a statement that the series $\left({ }^{2}\right)$

$$
I+(T-I) \lambda^{-1}+\left(T^{2}-T\right) \lambda^{-2}+\cdots+\left(T^{n}-T^{n-1}\right) \lambda^{-n}+\cdots
$$

is summable $(S)$ for $\lambda=1$. When the series converges, its sum is $(\lambda-1) R(\lambda)$ where $R(\lambda)$ is the resolvent of $T$,

$$
R(\lambda)=\sum_{n=0}^{\infty} T^{n} \lambda^{-n-1}
$$

Both series converge outside the least circle $|\lambda|=r$ which contains the spectrum of $T$.

The fundamental importance of the resolvent for all these questions has been brought out very clearly by Dunford. The present paper, which is an outgrowth of discussions between Professor Dunford and the author, is concerned with two aspects of the relationship between the properties of $R(\lambda)$ and the validity of an ergodic theorem for $T$.

The first aspect is the question of relations between different types of summability and corresponding ergodic theorems, in particular $(C, k)$ and Abel summability. By analogy with numerically valued series one would expect that the existence of

Presented to the Society, February 27, 1943, jointly with G. Szegö; received by the editors June 24, 1944.

(1) Numbers in brackets refer to the Bibliography at the end of the paper.

(2) In formulas (1.1) to (1.4) convergence and limits are taken in the sense of a fixed unspecified topology. 


$$
(C, k)-\lim T^{n}
$$

should imply the existence of

$$
\lim _{\lambda \rightarrow 1+0}(\lambda-1) R(\lambda)=(A)-\lim T^{n}
$$

and the equality of the two limits. Conversely, one might expect that the existence of the Abel limit should imply the existence of the $(C, k)$ limit provided appropriate Tauberian conditions are satisfied.

In the latter direction Dunford (oral communication, unpublished) has already extended the Fatou-Riesz theorem (see M. Riesz [13]). If $(\lambda-1) R(\lambda)$ is holomorphic at $\lambda=1$ where its value is $P$ and $\left\|T^{n}\right\| \leqq M$, then $(C, k)-\lim T^{n}$ $=P$ for $k>0$. In the present paper we extend $N$. Wiener's general Tauberian theorem (see [15, Theorem VIII]) and prove as a consequence that (A)-lim $T^{n}=P$ plus $\left\|T^{n}\right\| \leqq M$ implies $(C, k)$-lim $T^{n}=P$ for every $k>0$.

The second question concerns the character of $\lambda=1$ for the resolvent $R(\lambda)$ when an ergodic theorem holds for $T$. Dunford had shown (see [7, Theorem 3.16]) that if $(C, 1)-\lim T^{n}=P$ in the uniform topology, then $\|T\|=o(n)$ and $(\lambda-1) R(\lambda)$ is holomorphic and equals $P$ at $\lambda=1$. At the time when this investigation was started, Dunford had not yet considered the corresponding question in the strong topology and the first results (the case $\alpha=1$ of Theorems 10 and 11 below) showed that his conditions were no longer valid in the strong case. Though in the mean time Dunford [7, Theorem 3.19] has also settled the strong case, our results would still seem to be of some interest since they prove the existence of ergodic theorems of specified type for transformations which are of considerable interest in analysis, namely fractional integration.

In this paper a careful discussion is made of the transformations

$$
T_{\alpha} f=f(t)-\frac{1}{\Gamma(\alpha)} \int_{0}^{t}(t-u)^{\alpha-1} f(u) d u
$$

on the spaces $C_{0}[0,1]$ and $L_{1}(0,1)$. Here the resolvent $R_{\alpha}(\lambda)$ has $\lambda=1$ as its only singularity, but it is an essential singular point and not a pole. We prove the existence of $(A)-\lim T_{\alpha}^{n}$ for $0<\alpha<2$, of $(C, k)-\lim T_{1}^{n}$ for $k>\frac{1}{2}$, and of $\lim T_{\alpha}^{n}$ for $0<\alpha<1$, all in the strong topologies of the two spaces, and corresponding results for almost everywhere convergence when $f(t) \in L_{1}(0,1)$. The limits found for the parameters $\alpha$ and $k$ are sharp. Since, in particular, $(C, 1)-\lim T_{1}^{n}$ exists and $\left\|T_{1}^{n}\right\|$ is of the order of $n^{1 / 4}$, an example is given of a strong ergodic theorem in which the usual condition $\left\|T^{n}\right\| \leqq M$ for all $n$ is not satisfied. It is still a far cry from $O\left(n^{1 / 4}\right)$ to $o(n)$, however. For $\alpha=1$ the results follow from known properties of Laguerre polynomials, but for $\alpha \neq 1$ they require a detailed investigation of a class of polynomials which apparently has not been studied before. I am indebted to Professor G. Szegö for the asymptotic expressions of these polynomials presented in $\$ 7$ below. 
The subject matter is distributed as follows. Abstract Abelian and Tauberian theorems are given in $\$ 2$ with applications to ergodic theorems in $\$ 3$. That $\lambda=1$. need not be an isolated singularity of the resolvent in the strong topology is shown in $\S 4$. The discussion of $T_{\alpha}$ occupies $\S \S 5,6$, and 7 .

The present paper discusses relations between Tauberian theorems and spectral theory on one hand and ergodic theorems of the "discrete" type on the other. The "continuous" case may be subjected to a similar analysis, but here the discussion is more intimately connected with the analytic theory of semi-groups of linear transformations and will be given in another connection.

2. Some abstract Abelian and Tauberian theorems. In the present paragraph $X$ is a complex Banach space of elements $x, y, \cdots$ and convergence of a sequence $\left\{z_{n}\right\}$ to the limit $z_{0}$ is meant in the strong sense, that is $\left\|z_{n}-z_{0}\right\| \rightarrow 0$. We shall extend a few theorems of Abelian or Tauberian character to abstract spaces mainly with a view to relating $(C, k)$ and Abel summability.

THEOREM 1. Let $\left\{x_{n}\right\} \in X$ and let $k$ be fixed positive. Then

$$
(C, k)-\lim x_{n}=y_{0}
$$

implies that $\left(^{(3)}\right.$

$$
\begin{gathered}
\left\|x_{n}\right\|=o\left(n^{k}\right), \\
(A)-\lim x_{n}=y_{0} .
\end{gathered}
$$

We have to recall some formulas. Define $C_{n}^{(k)}$ by the generating function

so that

$$
(1-\mu)^{-1-k}=\sum_{n=0}^{\infty} C_{n}^{(k)} \mu^{n}
$$

$$
C_{n}^{(k)}=\left(\begin{array}{c}
n+k \\
n
\end{array}\right)=\frac{\Gamma(n+k+1)}{\Gamma(n+1) \Gamma(k+1)}
$$

and put

$$
C_{n}^{(k)} x_{n}^{(k)}=\sum_{\nu=0}^{n} C_{n-\nu}^{(k-1)} x_{\nu}
$$

Then (2.1) asserts that $x_{n}^{(k)} \rightarrow y_{0}$ when $n \rightarrow \infty$. Put

$$
\xi(\mu)=(1-\mu) \sum_{n=0}^{\infty} x_{n} \mu^{n}=(1-\mu)^{1+k} \sum_{n=0}^{\infty} C_{n}^{(k)} x_{n}^{(k)} \mu^{n} .
$$

Then (2.3) asserts that $\xi(\mu) \rightarrow y_{0}$ when $\mu \rightarrow 1-0$. For the proof of (2.3) it is enough to observe that

(3) Let us note in passing that (2.1) implies $(C, \kappa)$-lim $x_{n}=y$ for every $\kappa>k$. Proof as in the numerically valued case. This fact is used repeatedly below. 


$$
\left\|\xi(\mu)-y_{0}\right\| \leqq(1-\mu)^{1+k} \sum_{n=0}^{\infty} C_{n}^{(k)}\left\|x_{n}^{(k)}-y_{0}\right\| \mu^{n}
$$

and that $\left\{(1-\mu)^{1+k} C_{n}^{(k)} \mu^{n}\right\}$ is a limit preserving sequence of convergence factors. Hence from $\left\|x_{n}^{(k)}-y_{0}\right\| \rightarrow 0$ when $n \rightarrow \infty$ it follows that $\left\|\xi(\mu)-y_{0}\right\| \rightarrow 0$ when $\mu \rightarrow 1$. The proof given by S. Chapman [5, p. 379] for (2.2) in the case of numerically valued sequences can be followed step by step in the abstract case, merely replacing absolute values everywhere by norms.

We need the abstract form of Wiener's general Tauberian theorem $[15$, Theorem VIII].

Theorem 2. Let $K_{1}(u) \in L_{1}(-\infty, \infty)$ and suppose that

$$
(2 \pi)^{-1 / 2} \int_{-\infty}^{\infty} K_{1}(u) e^{-i t u} d u \neq 0
$$

for all real $t$. Let $z(u)$ be a bounded measurable $\left({ }^{4}\right)$ function on $(-\infty, \infty)$ to $X$ and suppose that

$$
\lim _{s \rightarrow \infty} \int_{-\infty}^{\infty} K_{1}(s-u) z(u) d u=z \int_{-\infty}^{\infty} K_{1}(u) d u,
$$

where the integral on the left is taken in the sense of Bochner [4] and $z \in X$. Let $K_{2}(u) \in L_{1}(-\infty, \infty)$. Then

$$
\lim _{s \rightarrow \infty} \int_{-\infty}^{\infty} K_{2}(s-u) z(u) d u=z \int_{-\infty}^{\infty} K_{2}(u) d u .
$$

We can follow Wiener's argument. It is clear that (2.8) implies

$$
\lim _{s \rightarrow \infty} \int_{-\infty}^{\infty}\left[\sum_{n=1}^{N} a_{n} K_{1}\left(s+\lambda_{n}-u\right)\right] z(u) d u=z \int_{-\infty}^{\infty}\left[\sum_{n=1}^{N} a_{n} K_{1}\left(u+\lambda_{n}\right)\right] d u
$$

for any choice of the complex constants $a_{n}$ and the real constants $\lambda_{n}$. But Wiener's basic theorem [15, Theorem II] asserts that (2.7) is a necessary and sufficient condition in order that the linear combinations of the translations of $K_{1}(u)$ be dense in $L_{1}(-\infty, \infty)$. We may consequently choose the constants so that $\left\|K_{2}(u)-\sum_{n=1}^{N} a_{n} K_{1}\left(u+\lambda_{n}\right)\right\|_{1}<\epsilon$. If, however, $\left\|K_{2}(u)-K_{3}(u)\right\|_{1}<\epsilon$ then for all real $s$

$$
\left\|\int_{-\infty}^{\infty}\left[K_{2}(s-u)-K_{3}(s-u)\right] z(u) d u\right\|<\epsilon \text { ess } \sup _{-\infty<u<\infty}\|z(u)\| .
$$

Formula (2.9) is a direct consequence of these estimates.

(4) That is, $z(u)$ is the limit for almost all $u$ of a sequence of finitely valued functions $z_{n}(u)$ such that the sets where $z_{n}(u)=x$ are (void or) measurable for all $x \in X$. 
We shall give only one application of this theorem, namely to the $[O-A \rightarrow(C, k)]$ theorem( $\left.{ }^{5}\right)$.

TheOREM 3. Let $\left\|x_{n}\right\| \leqq M$ for all $n$ and $(A)$-lim $x_{n}=y_{0}$. Then $(C, k)$ $\lim x_{n}=y_{0}$ for every positive $k$.

Let us define

$$
\begin{aligned}
& x_{k}(u)=x_{n}^{(k)} \text { for } n \leqq u<n+1, \quad n=0,1,2, \cdots, \\
& \Xi(\lambda)=\frac{\lambda^{1+k}}{\Gamma(1+k)} \int_{0}^{\infty} u^{k} e^{-\lambda u} x_{k}(u) d u,
\end{aligned}
$$

where the integral may be taken in the sense of Riemann-Graves. Since $\left\|x_{n}\right\| \leqq M$ we have also $\left\|x_{k}(u)\right\| \leqq M$ for all $u$. From (2.4) it follows that $\Gamma(k+1) C_{n}^{(k)}-n^{k}=O\left(n^{k-1}\right)$. It is then a simple matter to show that $\| \Xi(\lambda)$ $-\xi\left(e^{-\lambda}\right) \| \rightarrow 0$ when $\lambda \rightarrow+0$. Hence.

$$
\lim _{\lambda \rightarrow+0} \lambda^{1+k} \int_{0}^{\infty} u^{k} e^{-\lambda u} x_{k}(u) d u=y_{0} \Gamma(k+1) .
$$

This is a limit of Wiener's type if we take $\lambda=e^{-s}, u=e^{v}, K_{1}(v)=\exp [(1+k) v$ $\left.-e^{v}\right]$. Since

$$
\int_{-\infty}^{\infty} K_{1}(v) e^{-i t v} d v=\int_{0}^{\infty} e^{-u} u^{k-i t} d u=\Gamma(1+k-i t) \neq 0,
$$

condition (2.7) is satisfied. Let $h>0$ be fixed and define $K_{2}(v)$ to be $e^{-v}$ for $0 \leqq v \leqq \log (1+h)$ and zero outside of this interval. Formula (2.9) then gives after simplification

$$
\lim _{\omega \rightarrow \infty} \frac{1}{\omega h} \int_{\omega}^{\omega(1+h)} x_{k}(u) d u=y_{0}
$$

or

$$
y_{0}=\lim _{\omega \rightarrow \infty}\left\{x_{k}(\omega)+\frac{1}{\omega h} \int_{\omega}^{\omega(1+h)}\left[x_{k}(u)-x_{k}(\omega)\right] d u\right\} .
$$

In order to complete the proof we shall estimate the difference $\left[x_{k}(u)-x_{k}(\omega)\right]$ for $\omega \leqq u \leqq \omega(1+h)$. Using (2.5) we see that the required difference is of the form

$$
S=\left[C_{b}^{(k)}\right]^{-1} \sum_{\nu=0}^{b} C_{b-\nu}^{(k-1)} x_{\nu}-\left[C_{a}^{(k)}\right]^{-1} \sum_{\nu=0}^{a} C_{a-\nu}^{(k-1)} x_{\nu}
$$

There is no restriction in assuming that $\omega$ tends to infinity through integral

(5) Cf. A. F. Andersen $[1$, p. 80] and the remarks after the proof of Theorem 3. We give a direct proof based on the ideas of Wiener. 
values. We have then $\omega=[\omega]=a$ and $a<b<(1+h) a, b$ being also an integer. Hence $S=S_{1}+S_{2}$ where

$$
\begin{aligned}
& S_{1}=\sum_{\nu=0}^{a}\left\{C_{b-\nu}^{(k-1)}\left[C_{b}^{(k)}\right]^{-1}-C_{a-\nu}^{(k-1)}\left[C_{a}^{(k)}\right]^{-1}\right\} x_{\nu} \\
& S_{2}=\left[C_{b}^{(k)}\right]^{-1} \sum_{\nu=a+1}^{b} C_{b \rightarrow \nu}^{(k-1)} x_{\nu}
\end{aligned}
$$

It follows that

$$
\left\|S_{2}\right\| \leqq\left[C_{b}^{(k)}\right]^{-1} C_{b-a-1}^{(k)} M \leqq A M h^{k},
$$

where $A$ is a constant, possibly depending upon $k$ but not upon $a$ or $h$. In the case of $S_{1}$ we note that

$$
\frac{C_{n-\nu}^{(k-1)}}{C_{n}^{(k)}}=k \frac{\Gamma(n+1)}{\Gamma(n+1+k)} \frac{\Gamma(n-\nu+k)}{\Gamma(n-\nu+1)} .
$$

Here the first fraction on the right is a decreasing function of $n$ and if $k<1$; as we may suppose without restricting the generality $\left({ }^{\circ}\right)$, the second fraction is decreasing for $n>\nu$. Thus for $k<1$ the numerical coefficient of $x_{\nu}$ in $S_{1}$ is negative for $\nu=0,1, \cdots, a$. Hence

$$
\left\|S_{1}\right\| \leqq\left\{1-\left[C_{b}^{(k)}-C_{b-a-1}^{(k)}\right]\left[C_{b}^{(k)}\right]^{-1}\right\} M \leqq A M h^{k}
$$

and

$$
\left\|x_{k}(u)-x_{k}(\omega)\right\| \leqq 2 A M h^{k}, \quad \omega \leqq u \leqq \omega(1+h) .
$$

Thus, given any $\epsilon>0$ we can find an $\omega(\epsilon, h)$ such that for $\omega \geqq \omega(\epsilon, h)$

$$
\left\|x_{k}(\omega)-y_{0}\right\| \leqq \epsilon+2 A M h^{k} .
$$

From this we conclude that $x_{k}(\omega) \rightarrow y_{0}$ when $\omega \rightarrow \infty$ and the theorem is proved.

At the end of his paper on the Abel $\rightarrow$ Cesàro problem, J. E. Littlewood $[10$, p. 448] remarks that a series is $(C, 1)$ summable if its partial sums are bounded and it is summable Abel. That such a series is actually summable $(C, k)$ for every $k>0$ was first proved by A. F. Andersen $[1, \mathrm{p} .80]$ as a special case of a more general theorem. The latter can also be extended to Banach spaces. We state the result without proof.

THEOREM 4. If $\left\{x_{n}\right\} \in X$ and $(A)-\lim x_{n}=y_{0}$ and, in addition, $\left\|x_{n}^{(2)}\right\| \leqq M$, then $(C, k+\delta)$-lim $x_{n}=y_{0}$ for every $\delta>0$.

The theorem of Andersen is also an extension of an earlier result of Littlewood (loc. cit.) according to which a series which is finite $(C, r)$ and summable Abel is summable $(C, r+1)$.

(') Cf. footnote 3. 
On the other hand, from the assumption that $(A)$-lim $x_{n}$ exists and $\left\|x_{n}\right\| \leqq M n^{\gamma}$ for some fixed $\gamma>0$ it is not possible to conclude that $(C, k)$-lim $x_{n}$ exists for any $k$. Indeed, Littlewood (loc. cit.) gives an example of an Abel summable series, the partial sums of which are $O\left[(\log n)^{1+\varepsilon}\right]$, which is not summable $(C)$.

The above theorems can be extended in various other directions, however. An important case is that in which the elements $x_{n}$ are functions of a numerical parameter $\xi$ defined on a measurable set $S, x_{n}=x_{n}(\xi), \xi \in S$. The assumptions regarding boundedness and convergence in the theorems may then depend upon $\xi$ and as a consequence the conclusions will also depend upon $\xi$. We note three cases of some interest.

(1) If the assumptions hold uniformly with respect to $\xi$, so do the conclusions.

(2) If the assumptions hold for almost all $\xi$, so do the conclusions.

(3) If in the assumptions the bounds are integrable functions of $\xi$ and convergence holds in measure with respect to $\xi$, the same is true in the conclusions.

We recall that $y_{n}(\xi)$ converges to $y_{0}(\xi)$ in measure (or asymptotically) with respect to $\xi$ in $S$ if to every given $\epsilon>0$ the measure of the subset of $S$ where $\left\|y_{n}(\xi)-y_{0}(\xi)\right\|>\epsilon$ tends to zero when $n \rightarrow \infty$.

3. Applications to ergodic theorems. In the following $X$ is the basic and $Z$ an auxiliary Banach space which will be identified with $X$ or related spaces later. The space of all linear bounded operators on $X$ to $X$ is denoted by $X$. Finally $E_{2}$ is the space of complex numbers with the usual metric. A combination of Theorems 1 and 3 gives the following theorem.

THEOREM 5. Let $\left\{z_{n}\right\}$ be a sequence of elements in a complex Banach space $Z$. Let

$$
R(\lambda ; z)=\sum_{n=0}^{\infty} z_{n} \lambda^{-n-1},
$$

when the series converges $\left({ }^{7}\right)$. A necessary condition in order that $\left({ }^{8}\right)$

$$
\lim _{n \rightarrow \infty}\left[C_{n}^{(k)}\right]^{-1} \sum_{\nu=0}^{n} C_{n \rightarrow \nu}^{(k-1)} z_{\nu}=z
$$

for some fixed positive $k$ is that

$$
\begin{gathered}
\lim _{\lambda \rightarrow 1+0}(\lambda-1) R(\lambda ; z)=z, \\
\lim _{n \rightarrow \infty} n^{-k} z_{n}=0 .
\end{gathered}
$$

(7) The series converges for $|\lambda|>1$ if (3.2) holds.

( $\left.{ }^{8}\right)$ More concisely expressed: $a(C, k)$-ergodic sequence $\left\{z_{n}\right\}$ is $(A)$-ergodic and a bounded $(A)$-ergodic sequence is $(C, k)$-ergodic for every $k>0$. It is obvious that similar results hold for much more general definitions of summation than that of Cesàro. 
Conversely, if (3.3) holds and (3.4) is replaced by $\left\|z_{n}\right\| \leqq M$ for all $n$, then (3.2) holds for every positive $k$.

Ergodic theorems are obtained by suitable specialization. Let us first take $Z=X, z_{0}=I, z_{1}=T, \cdots, z_{n}=T^{n}, \cdots$, where $T \in X$. Then

$$
R(\lambda ; z)=R(\lambda ; T)=\sum_{n=0}^{\infty} T^{n} \lambda^{-n-1}
$$

is the resolvent of $T$ and we obtain a "uniform" ergodic theorem.

ThEOREM 6. A necessary condition for the existence of a $P \in X$ such that for some fixed $k>0$

$$
\lim _{n \rightarrow \infty}\left[C_{n}^{(k)}\right]^{-1} \sum_{\nu=0}^{n} C_{n-\nu}^{(k-1)} T^{\nu}=P
$$

is that

$$
\begin{gathered}
\lim _{\lambda \rightarrow 1+0}(\lambda-1) R(\lambda ; T)=P, \\
\lim _{n \rightarrow \infty} n^{-k} T^{n}=0 .
\end{gathered}
$$

Conversely, if (3.7) holds but (3.8) be replaced by $\left\|T^{n}\right\| \leqq M$ for all $n$, then (3.6) holds for every $k>0$. Here convergence is taken in the sense of the uniform topology of $X$.

The same theorem holds if $X$ is an arbitrary commutative normed ring.

We get a "strong" or "mean" ergodic theorem by taking $Z=X, z_{0}=x$, $z_{1}=T x, \cdots, z_{n}=T^{n} x, \cdots, x \in X, T \in X$.

THEOREM 7. A necessary condition for the existence of a $P \in X$ such that for some fixed $k>0$ and all $x \in X$

$$
\lim _{n \rightarrow \infty}\left[C_{n}^{(k)}\right]^{-1} \sum_{\nu=0}^{n} C_{n-\nu}^{(k-1)} T^{\nu} x=P x
$$

is that for all $x$

$$
\lim _{\lambda \rightarrow 1+0}(\lambda-1) R(\lambda ; T) x=P x
$$

and

$$
\lim _{n \rightarrow \infty} n^{-k} T^{n} x=0 .
$$

Conversely, if (3.10) holds and if (3.11) be replaced by $\left\|T^{n}\right\| \leqq M$ for all $n$, then (3.9) holds for every $k>0$. Convergence in (3.9) and (3.10) is taken in the sense of the strong topology of $X$. 
We omit the case of "weak" ergodic theorems and pass over to theorems of "almost everywhere" type ("strong" theorems in the terminology of G. D. Birkhoff [3]). Here we disregard the case contemplated at the end of $\$ 2$ where the norm is a function of $\xi$. Let $X$ be a complex Banach space the elements of which are complex-valued measurable functions $x(\xi)$ on a set $S$ in a euclidean space. We take $Z=E_{2}, z_{0}=x(\xi), z_{1}=T x(\xi), \cdots, z_{n}=T^{n} x(\xi), \cdots$, where $T \in X$.

THEOREM 8. A necessary condition for the existence of a transformation $P \in X$ such that for a fixed $k>0$

$$
\lim _{n \rightarrow \infty} \sigma_{n}^{(k)}[T] x(\xi) \equiv \lim _{n \rightarrow \infty}\left[C_{n}^{(k)}\right]^{-1} \sum_{\nu=0}^{n} C_{n-\nu}^{(k-1)} T^{\nu} x(\xi)=P x(\xi)
$$

for all $x(.) \in X$ and for almost all $\xi$ (where the exceptional set may depend upon $x()$.$) is that$

$$
\begin{gathered}
\lim _{\lambda \rightarrow 1+0}(\lambda-1) R(\lambda ; T) x(\xi)=P x(\xi), \\
\lim _{n \rightarrow \infty} n^{-k} T^{n} x(\xi)=0
\end{gathered}
$$

for all $x($.$) and almost all \xi$. If (3.13) holds but (3.14) be replaced by $\left|\sigma_{n}^{(\beta)}[T] x(\xi)\right|$ $\leqq M(\xi)$ for all $n$, where $\beta \geqq 0$ and $M(\xi)$ is a measurable function finite almost everywhere, then (3.12) holds for all $k>\beta$. Convergence is taken in the classical, pointwise sense $\left(^{(9)}\right.$.

In the converse part we have used Theorem 4 instead of Theorem 3 to obtain greater generality. Similar extensions hold for Theorems 5, 6, and 7 .

4. The non-polar character of $\lambda=1$ for "strong" resolvents. A sufficient condition for the validity of (3.3) in Theorem 5 is that $R(\lambda ; z)$ have a simple pole at $\lambda=1$ of residue $z$. Dunford [7, Theorem 3.16] could show in the "uniform" case that (3.6) implies not merely (3.7) but also the much stronger conclusion that $\lambda=1$ is a simple pole of $R(\lambda ; T)$ of residue $P\left({ }^{10}\right)$. We shall show that this is no longer true in the "strong" case.

Our first example is taken from the theory of semi-groups of linear selfadjoint bounded transformations on a Hilbert space to itself (see E. Hille [8, $\$ 4.1]$, where further references to the literature are to be found). The transformation $T_{s}$ is defined for $s>0$ and the semi-group property $T_{s} T_{t}=T_{s+t} \mathrm{im}$ plies that $T_{s}$ is positive definite. Consequently its spectrum $S\left(T_{s}\right)$ is real positive. If we impose the restriction

( 9 ) We are tacitly assuming that the definitions of addition, scalar multiplication, and convergence for the elements $x($.$) of X$ are consistent with those holding in $E_{2}$ for the point functions $x(\sigma)$. It is not necessary that $S$ be a subset of a euclidean space or that measurability be taken in the sense of Lebesgue. We do not insist further on such refinements, however.

(10) If $\lambda=1$ should belong to the resolvent set instead of to the spectrum, $P=0$. Assumption (3.7) does not figure explicitly in Dunford's theorem, but it is usually implied by his assumption that the operator $T$ is ergodic in a specified sense. 


$$
\| T \_\leqq 1 \text {, }
$$

then $S\left(T_{s}\right)$ will be restricted to the interval $(0,1)$ of the real axis in the $\lambda$-plane and $T_{\infty} x$ is represented by a Laplace-Stieltjes integral

$$
T, x=\int_{0}^{\infty} e^{-s u} d E(u) x .
$$

Here the integral converges for $\Re(s) \geqq 0$ and defines a semi-group of linear bounded transformations, not merely for real positive $s$, but in the whole half-plane of convergence. The self-adjoint character is of course lost for complex $s$, but $T_{s}$ and $T_{\bar{s}}$ are adjoint transformations. The transformation $E(u)$ is the resolution of the identity of a positive definite self-adjoint transformation

$$
B=\lim _{h \rightarrow \infty} \frac{1}{h}\left[I-T_{h}\right]
$$

where the limit is taken in the strong sense and the domain of $B$ is dense in the space. We normalize $E(u)$ by assuming it to be strongly left-continuous (11), that is $E(u-h) x \rightarrow E(u) x$ when $h \rightarrow+0, u$ being fixed positive. Further $E(u) E(v)=E(w), w=\min (u, v), E(u)=0$ for $u \leqq 0$ and $E(u) x \rightarrow x$ when $u \rightarrow+\infty$. From (4.2) we can compute the resolvent of $T$, and find

$$
R\left(\lambda, T_{s}\right) x=\int_{0}^{\infty} \frac{d E(u) x}{\lambda-e^{-s u}}
$$

for all $\lambda$ not in $S\left(T_{s}\right)$. A point $\lambda_{0}$ of the interval $(0,1)$ belongs to the resolvent set of $T_{s}$ if and only if $u_{0}=(1 / s) \log \left(1 / \lambda_{0}\right)$ lies in an interval of constancy of $E(u)$. On the other hand, $\lambda_{0}$ belongs to the point spectrum of $T$, if and only if $\left[E\left(u_{0}+0\right)-E\left(u_{0}\right)\right] x$ is not identically zero. A simple calculation shows that

$$
\left(\lambda-\lambda_{0}\right) R\left(\lambda ; T_{s}\right) x \rightarrow\left[E\left(u_{0}+0\right)-E\left(u_{0}\right)\right] x, \quad u_{0}=\frac{1}{s} \log \frac{1}{\lambda_{0}},
$$

when $\lambda \rightarrow \lambda_{0}$ in the sectors $0<\epsilon \leqq\left|\arg \left(\lambda-\lambda_{0}\right)\right| \leqq \pi-\epsilon$. In particular,

$$
(\lambda-1) R\left(\lambda ; T_{s}\right) x \rightarrow E(+0) x
$$

and here $\lambda$ may approach 1 throughout the sector $|\arg (\lambda-1)| \leqq \pi-\epsilon$.

Formulas (4.1) and (4.4) show that the assumptions of Theorem 7 are satisfied. Since $T_{s}^{n}=T_{n s}$, we have consequently

$$
(C, k)-\lim T_{n s} x=E(+0) x, \quad k>0,
$$

(i1) The customary normalization requires strong right semi-continuity. If this convention should be adopted here, it would become necessary to replace the lower limit 0 in formula (4.2) by an arbitrary negative quantity and to make similar adjustments in the other formulas of this paragraph. 
for all $x$. On the other hand, $\lambda=1$ is a pole of $R\left(\lambda ; T_{\bullet}\right)$ if and only if $E(u)$ is constant for small positive $u$ and has a saltus at $u=0$, that is, there exists a $\delta>0$ such that $E(u)=E(+0) \neq E(0)=0$ for $0<u \leqq \delta$. If there is no such interval of constancy of $\dot{E}(u)$, then $\lambda=1$ is not an isolated singularity. This is to be regarded as the general case, since the semi-group $T_{\mathrm{a}}$ is defined as soon as $E(u)$ is given subject to the conditions stated above which do not involve any assumptions of constancy on an interval $(0, \delta)$ and do not imply any such restriction.

Actually (4.5) holds also for $k=0$, that is,

$$
\lim _{n \rightarrow \infty} T_{n s} x=\lim _{\omega \rightarrow \infty} T_{\omega} x=E(+0) x .
$$

This follows from a well known property of Laplace-Stieltjes integrals according to which the limit of the integral for $s \rightarrow+\infty$ is the saltus of the integrator at the origin. This property also holds for vector-valued integrals of the type considered here. We have consequently proved the following "mean" ergodic theorem.

THEOREM 9. If $\left\{T_{0}\right\}, s>0$, is a semi-group of linear self-adjoint transformations on a Hilbert space satisfying (4.1), then (4.6) holds for all $x$.

It should be observed that the corresponding theorem for groups of unitary transformations is false. This can be seen by considering, for instance, the group of translations in an $L_{2}$-space such that $U_{s} x(t)=x(t+s)$ where $x(t) \in L_{2}(a, b)$ and is continued periodically outside $(a, b)$ if $b-a$ is finite and by symmetry if $(a, b)$ extends to infinity one way only. For groups of unitary transformations on Hilbert space we have the quasi-ergodic theorem of $\mathrm{J}$. von Neumann [11] which asserts, among other things, that $(C, 1)$-lim $U_{\alpha}^{n}$ exists in the strong sense and is a projection $P_{\alpha}$. Our Theorem 7 shows that the stronger assertion( $\left.{ }^{12}\right)(C, k)$-lim $U_{\alpha}^{n}=P_{\alpha}$ holds for every $k>0$, and here, as we have just remarked, $k$ cannot be allowed to be zero.

The rest of the paper will be devoted to a discussion of ergodic theorems relating to the operation of fractional integration in the Riemann-Liouville sense.

5. (A)-ergodic theorems relating to fractional integration. We consider simultaneously the two function spaces $C_{0}[0,1]$ and $L_{1}(0,1)$. The first is the space of functions $f(t)$ continuous for $0 \leqq t \leqq 1$ such that $f(0)=0$ with $\|f\|=\max |f(t)|$, the latter the space of integrable functions on $(0,1)$ with the usual metric. We note that the elements of $C_{0}[0,1]$ are dense in $L_{1}(0,1)$.

Let $\alpha$ be fixed positive and define

$$
J_{\alpha}[f]=\frac{1}{\Gamma(\alpha)} \int_{0}^{t}(t-u)^{\alpha-1} f(u) d u, \quad 0 \leqq t \leqq 1,
$$

(12) This is included in results of L. W. Cohen [6, p. 508], however, since his assumptions are obviously satisfied in the present case. 


$$
T_{\alpha}[f]=\left(I-J_{\alpha}\right) f .
$$

These are obviously linear bounded transformations on the spaces in question. The norm of $J_{\alpha}$ does not exceed $[\Gamma(\alpha+1)]^{-1}$ in either space. We recall that $J_{\alpha} J_{\beta}=J_{\alpha+\beta}$, in particular, $J_{\alpha}^{n}=J_{n \alpha}$. We shall study the iterates of $T_{\alpha}$. A simple calculation gives

$$
\begin{aligned}
T_{\alpha}^{n}[f] & =f(t)-\int_{0}^{t} P_{n}(t-u, \alpha) f(u) d u, \\
P_{n}(w, \alpha) & =\sum_{\nu=1}^{n}(-1)^{\nu-1}\left(\begin{array}{l}
n \\
\nu
\end{array}\right) \frac{w^{\nu \alpha-1}}{\Gamma(\nu \alpha)} .
\end{aligned}
$$

For $\alpha=1$ the latter expression is a Laguerre polynomial in $w$ (for notation and properties see G. Szegö [14, chapters V, VIII])

$$
P_{n}(w, 1)=L_{n-1}^{(1)}(w) \text {. }
$$

In the present paragraph we shall be concerned with $(A)$-ergodic properties of $T_{\alpha}$ of the strong or almost everywhere types, that is relations like (3.10) and (3.13) above, and start by computing the resolvent $R\left(\lambda, T_{\alpha}\right)$ $\equiv R_{\alpha}(\lambda)$. For the following compare E. Hille and J. D. Tamarkin $[9$, pp. 524525] and E. Hille $\left[8\right.$, p. 43]. For $|\lambda-1|>\left\|J_{\alpha}\right\|$ we have

Hence

$$
\begin{aligned}
R_{\alpha}(\lambda) & =\left[\lambda I-T_{\alpha}\right]^{-1}=\left[(\lambda-1) I+J_{\alpha}\right]^{-1} \\
& =(\lambda-1)^{-1}\left[I+(\lambda-1)^{-1} J_{\alpha}\right]^{-1} \\
& =(\lambda-1)^{-1} I+(\lambda-1)^{-1} \sum_{n=1}^{\infty}(1-\lambda)^{-n} J_{n \alpha} .
\end{aligned}
$$

$$
R_{\alpha}(\lambda) f=(\lambda-1)^{-1} f(t)+(\lambda-1)^{-1} \int_{0}^{t} \sum_{n=1}^{\infty} \frac{(t-u)^{n \alpha-1}}{\Gamma(n \alpha)(1-\lambda)^{n}} f(u) d u .
$$

This representation is evidently valid for all values of $\lambda \neq 1$ and not merely for $|\lambda-1|>\left\|J_{\alpha}\right\|$. Thus $R_{\alpha}(\lambda) f$ is an entire function of $1 /(\lambda-1)\left({ }^{13}\right)$. The kernel

$$
K_{\alpha}(w, \lambda)=\sum_{n=1}^{\infty} \frac{w^{n \alpha-1}}{\Gamma(n \alpha)(1-\lambda)^{n}}
$$

is evidently expressible in terms of Mittag-Leffler's function $E_{\alpha}(z)$ as

$$
K_{\alpha}(w, \lambda)=\frac{d}{d w} E_{\alpha}\left(\frac{w^{\alpha}}{1-\lambda}\right) .
$$

(12) The spectrum of $T_{\alpha}$ consists of one single point, $\lambda=1$, which, nevertheless, makes up the "continuous" spectrum. To see this, we note that the functions $f_{n}=t^{n}$ have norm 1 in $C_{0}[0,1]$ but $\left(I-T_{\alpha}\right) f_{n}=J_{\alpha} f_{n}$ converges strongly to zero when $n \rightarrow \infty$. In $L_{1}(0,1)$ we take $f_{n}=(n+1) t^{n}$ instead. 
In discussing the ergodic properties of $T_{\alpha}$ we shall need a couple of lemmas concerning singular integrals. The proofs follow classical lines and are omitted here.

LEMMA 1. Let $K_{\nu}(w) \in L_{1}(0,1)$ for all $\nu$ and be such that

$$
\int_{0}^{t} K_{\nu}(w) d w \rightarrow 1, \quad K_{v}(t) \rightarrow 0, t>0,
$$

when $\nu \rightarrow \infty$, both relations holding uniformly with respect to $t$ in $0<\epsilon \leqq t \leqq 1$ for every $\epsilon>0$, and

$$
\int_{0}^{1}\left|K_{\nu}(w)\right| d w \leqq M \text { for all } \nu .
$$

Let $f(t) \in C_{0}[0,1]$. Then

$$
f_{\nu}(t) \equiv \int_{0}^{t} K_{\nu}(t-u) f(u) d u \rightarrow f(t)
$$

when $\nu \rightarrow \infty$, uniformly in $t, 0 \leqq t \leqq 1$, that is, $f_{\nu}(t)$ converges strongly to $f(t)$.

Lemma 2. Let $K_{\nu}(w) \in L_{1}(0,1)$ for all $\nu$ and satisfy the conditions of Lemma 1, but not necessarily uniformly with respect to $t$ in (i). In addition, let there exist a dominant $G_{\nu}(w)$ such that $\left|K_{\nu}(w)\right| \leqq G_{\nu}(w)$ for all $\nu$ and $w$. Here $G_{\nu}(w)$ shall be a positive, monotone decreasing function of $w$ for fixed $\nu$ which is absolutely continuous on $(\epsilon, 1)$ for every $\epsilon>0$. Further, $G_{\nu}(w)$ shall satisfy condition (ii) and for fixed $w>0, G_{\nu}(w) \rightarrow 0$ when $\nu \rightarrow \infty$. If $f(t) \in L_{1}(0,1)$ then $f_{\nu}(t)$ exists for almost all $t, f_{\nu}(t) \in L_{1}(0,1)$, and $f_{\nu}(t) \rightarrow f(t)$ when $\nu \rightarrow \infty$ for almost all $t$.

We shall now prove the following theorem.

THEOREM 10. Let $\alpha$ be fixed, $0<\alpha<2$. Then

$$
(\lambda-1) R_{\alpha}(\lambda) f \rightarrow 0 \text { when } \lambda \rightarrow 1
$$

for every $f \in C_{0}[0,1]$ or $L_{1}(0,1)$ in the sense of strong convergence in the space in question. Here $\lambda$ may tend to 1 radially $\left.{ }^{14}\right)$ in the sector $|\arg (\lambda-1)|<(2-\alpha) \pi / 2$. If $f(t) \in L_{1}(0,1),(5.9)$ also holds in the sense of pointwise convergence for almost all $t$. The theorem becomes false for $\alpha \geqq 2$.

By virtue of formulas (5.6) and (5.7) we have

$$
(\lambda-1) R_{\alpha}(\lambda) f=f(t)+\int_{0}^{t} K_{\alpha}(t-u, \lambda) f(u) d u,
$$

and we shall prove that the kernel $-K_{\alpha}(w, \lambda)$ satisfies the conditions of Lemmas 1 and 2 . For this purpose we shall need the following classical for-

(14) More generally, along any Stolz' path. 
mulas $\left({ }^{15}\right)$ from the theory of the function $E_{\alpha}(z)$ which are valid when $0<\alpha<2$ :

$$
E_{\alpha}(z)=\frac{1}{\alpha} \exp \left[z^{1 / \alpha}\right]+o(1), \quad|\arg z|<\alpha \frac{\pi}{2},
$$

$$
\left.\begin{array}{l}
E_{\alpha}(z)=-\frac{1}{z \Gamma(1-\alpha)}+o\left(\frac{1}{z^{2}}\right) \\
E_{\alpha}^{\prime}(z)=\frac{1}{z^{2} \Gamma(1-\alpha)}+o\left(\frac{1}{z^{3}}\right)
\end{array}\right\}, \quad \alpha \frac{\pi}{2}<\arg z<(4-\alpha) \frac{\pi}{2}
$$

Using (5.8) and (5.12) we see that

$$
-\int_{0}^{t} K_{\alpha}(w, \lambda) d w=1-E_{\alpha}\left(\frac{t^{\alpha}}{1-\lambda}\right) \rightarrow 1
$$

when $\lambda \rightarrow 1$ radially in the sector $|\arg (\lambda-1)|<(2-\alpha) \pi / 2$. The convergence is obviously uniform with respect to $t$ in any interval $(\epsilon, 1), \epsilon>0$, and also uniform with respect to $\lambda$ in any fixed interior sector. Formulas (5.8) and (5.13) show that $\lim _{\lambda \rightarrow 1} K_{\alpha}(w, \lambda)=0$, uniformly in $0<\epsilon \leqq w \leqq 1$. Thus condition (i) of Lemma 1 is satisfied.

Let $\epsilon>0$ be fixed and $(\alpha+\epsilon) \pi / 2 \leqq \theta \leqq(4-\alpha-\epsilon) \pi / 2$. Formula (5.13) shows that $E_{\alpha}(z)$ is of bounded variation on every ray arg $z=\theta$ and the total variation is less than some fixed quantity $B_{\epsilon}$, that is

$$
\int_{0}^{\infty}\left|E_{\alpha}^{\prime}\left(r e^{i \theta}\right)\right| d r \leqq B \text {. }
$$

But if $\arg (\lambda-1)=\pi-\theta$ then

$$
\int_{0}^{1}\left|K_{\alpha}(w, \lambda)\right| d w=\int_{0}^{\Delta(\lambda)}\left|E_{\alpha}^{\prime}\left(r e^{i \theta}\right)\right| d r \leqq B_{e},
$$

where $A(\lambda)=\left.|\lambda-1|\right|^{-1}$. Hence condition (ii) is also satisfied and uniformly with respect to $\lambda$ in the sector $|\arg (\lambda-1)| \leqq(2-\alpha-\epsilon) \pi / 2$. It follows that the singular integral in (5.10) satisfies the conditions of Lemma 1 and consequently (5.9) holds in the sense of strong convergence in $C_{0}[0,1]$. Moreover, the convergence is uniform with respect to $\lambda$ in any interior sector $\left({ }^{16}\right)$.

(16) See, for example, A. Wiman [16, formula (5)] from which (5.11) and (5.12) can be read off. Differentiation of (5) gives (5.13).

(16) On the other hand, (5.9) cannot hold in the uniform topology. This is not difficult to show directly, but for $0<\alpha \leqq 1$ the contrary assumption would lead to a contradiction with Dunford [7, Theorem 3.16]. Indeed, this assumption by virtue of formula (6.5) below together with our Theorem 6 would show that the sequence $T_{\alpha}^{n}$ had to be $(C)$-ergodic in the uniform topology and this would imply that $\lambda=1$ were at most a simple pole of $R_{\alpha}(\lambda)$ which is obviously false. 
Formula (5.15) shows that for $|\arg (\lambda-1)| \leqq(2-\alpha-\epsilon) \pi / 2$

$$
\left\|(\lambda-1) R_{\alpha}(\lambda)\right\| \leqq 1+B_{e},
$$

where it is immaterial if the norm is taken in $C_{0}[0,1]$ or in $L_{1}(0,1)$. But the elements of the former space are dense in the latter, and strong convergence of a sequence of elements in $C_{0}[0,1]$ implies strong convergence of the same sequence in $L_{1}(0,1)$. Formula (5.16) then implies that (5.9) holds for every $f \in L_{1}$, in the sense of strong (=mean) convergence, by the Hahn-BanachSteinhaus theorem (see $[2$, p. 79]).

We now proceed to the question of pointwise almost everywhere convergence when $f \in L_{1}$. In order to apply Lemma 2 we have merely to determine a suitable dominant $G_{\alpha}(w, \lambda)$ of $K_{\alpha}(w, \lambda)$. Formula (5.13) shows, however, that we can determine a $C=C(\alpha)$ such that if $|\lambda-1|<1,|\arg (\lambda-1)|$ $\leqq(2-\alpha-\epsilon) \pi / 2$, and

$$
G_{\alpha}(w, \lambda)=\left\{\begin{array}{l}
C(\alpha)|\lambda-1|^{-1 / \alpha}, 0 \leqq w<|\lambda-1|^{1 / \alpha}, \\
C(\alpha)|\lambda-1| w^{-\alpha-1},|\lambda-1|^{1 / \alpha} \leqq w \leqq 1,
\end{array}\right.
$$

then $\left|K_{\alpha}(w, \lambda)\right| \leqq G_{\alpha}(w, \lambda)$ and all the conditions of Lemma 2 are satisfied. Hence $(\lambda-1) R_{\alpha}(\lambda) f(t) \rightarrow 0$ for almost all $t$ when $\lambda \rightarrow 1$ in the sector indicated.

It remains to settle the case $\alpha \geqq 2$. For $\alpha=2$ we have

$$
(\lambda-1) R_{2}(\lambda) f=f(t)-(\lambda-1)^{-1 / 2} \int_{0}^{t} \sin \left[(\lambda-1)^{-1 / 2}(t-u)\right] f(u) d u
$$

and for $\lambda>1$

$$
\int_{0}^{1}\left|K_{2}(w, \lambda)\right| d w=\frac{2}{\pi}(\lambda-1)^{-1 / 2}+O(1) .
$$

It is not difficult to see that the norm of $(\lambda-1) R_{2}(\lambda)$ in $C_{0}[0,1]$ as well as in $L_{1}(0,1)$ is of the same order of magnitude, that is, tends to infinity when $\lambda \rightarrow 1$. This excludes the possibility of (5.9) being true in the sense of strong convergence when $\alpha=2$. The situation is still more unfavorable when $\alpha>2$, because then $E_{\alpha}(z)$ and $E_{\alpha}^{\prime}(z)$ are unbounded on every ray $\left({ }^{17}\right)$. Both functions are oscillatory on the negative real axis in such a manner that amplitudes as well as frequencies tend to infinity with $|z|$. From this we conclude that lim sup $\left\|(\lambda-1) R_{\alpha}(\lambda)\right\|=\infty$ when $\lambda \rightarrow 1$ and strong convergence is out of question.

Pointwise convergence still remains as a possibility. We note, however, that (5.14) may be rewritten as

$$
(\lambda-1) R_{\alpha}(\lambda) \cdot 1=E_{\alpha}\left(\frac{t^{\alpha}}{1-\lambda}\right) .
$$

(17) See A. Wiman [16, pp. 220-221]. 
If $t=0$, the right-hand side always equals one, but if $0<t \leqq 1$ and $2 \leqq \alpha$, the right-hand side never tends to a limit when $\lambda \rightarrow 1+0$. Thus there exists at least one function of $L_{1}(0,1)$ for which $(\lambda-1) R_{\alpha}(\lambda) f(t)$ does not converge pointwise when $\lambda \rightarrow 1+0$ for any value of $\alpha \geqq 2$. This completes the proof of Theorem 10.

6. (C)-ergodic theorems relating to fractional integration. Here our main result is the following.

THEOREM 11. The transformation $T_{\alpha}$ of formula (5.2) is strongly $(C)$-ergodic in $C_{0}[0,1]$ and $L_{1}(0,1)$ when $0<\alpha \leqq 1$ but never when $\alpha>1$. If $0<\alpha<1$, then $\left\|T_{\alpha}^{n}\right\|$ is bounded for all $n$ and $T_{\alpha}^{n} f$ tends strongly to zero for every $f(t)$ in $C_{0}[0,1]$ or in $L_{1}(0,1)$. Further, $T_{\alpha}^{n} f(t) \rightarrow 0$ for almost all $t$ for every $f(t) \in L_{1}(0,1)$. If $\alpha=1,\left\|T_{\alpha}^{n}\right\|=O\left(n^{1 / 4}\right)$ and $(C, k)-\lim T_{\alpha}^{n} f=0$ for every $k>\frac{1}{2}$ but for no $k \leqq \frac{1}{2}$, convergence holding in the strong sense in both spaces. For $k>\frac{1}{2}$ we have also pointwise convergence to zero for almost all $t$. When $\alpha>1$ we have $\log \left\|T_{\alpha}^{n}\right\|$ $>C(\alpha) n^{1 /(\alpha+1)}$ and no $(C)$-ergodic theorems are possible.

For the proof we refer back to formula (5.3). Let $\alpha$ be fixed, $0<\alpha<1$. It is sufficient to prove that the kernel $P_{n}(w, \alpha)$ satisfies the conditions of Lemmas 1 and 2. We start by proving that for $0<t \leqq 1$

$$
\int_{0}^{t} P_{n}(w, \alpha) d w \rightarrow 1 \text { when } n \rightarrow \infty
$$

uniformly with respect to $t$ in any interval $(\epsilon, 1)$. For this purpose we evaluate $(\lambda-1) R_{\alpha}(\lambda) \cdot 1$ anew. One expression of this function was given in formula (5.17). Another follows from computing the sum of the series $\sum_{0}^{\infty} \lambda^{-n-1} T_{\alpha}^{n} \cdot 1$. Equating the two expressions we obtain the identity

$$
(\lambda-1) \sum_{n=0}^{\infty} \lambda^{-n-1} \int_{0}^{t} P_{n}(w, \alpha) d w=1-E_{\alpha}\left(\frac{t^{\alpha}}{1-\lambda}\right)
$$

and

$$
\int_{0}^{t} P_{n}(w, \alpha) d w=1-\frac{1}{2 \pi i} \int_{C} \lambda^{n}(\lambda-1)^{-1} E_{\alpha}\left(\frac{t^{\alpha}}{1-\lambda}\right) d \lambda,
$$

where to start with the contour of integration may be taken as the circle $|\lambda|=r>1$. This contour, however, may be deformed into one inside the unit circle. Let $\Gamma_{n}$ be the circle $|\lambda|=r_{n}<1$ and draw the two tangents to $\Gamma_{n}$ from $\lambda=1$. If $r_{n}$ is sufficiently near to 1 the tangents will make angles with the positive real axis which are numerically less than $(2-\alpha) \pi / 2$. The new contour of integration $C_{n}$ will then consist of the two tangents plus the major arc of the circle $\Gamma_{n}$ between the points of tangency. By virtue of formula (5.12) the integrand is uniformly bounded on $C_{n}$; the contributions to the value of the integral from the rectilinear and the circular parts of the path are 
$O\left[\left(1-r_{n}\right)^{1 / 2}\right]$ and $O\left(r_{n}^{n}\right)$ respectively. Choosing $r_{n}=1-n^{-1} \log n$ we see that the integral tends to zero when $n \rightarrow \infty$. This proves (6.2).

Formulas (7.4) and (7.11) below show that for a fixed $\alpha, 0<\alpha<1$, positive constants $A=A(\alpha)$ and $C=C(\alpha)$ can be found such that

$$
\left|P_{n}(w, \alpha)\right| \leqq\left\{\begin{array}{lr}
A n w^{\alpha-1}, & 0<n w^{\alpha} \leqq 1, \\
n^{1 / \alpha} \exp \left[-C\left(n w^{\alpha}\right)^{1 /(\alpha+1)}\right], & 1<n w^{\alpha}, w \text { bounded. }
\end{array}\right.
$$

A simple calculation shows that this estimate implies

$$
\int_{0}^{1}\left|P_{n}(w, \alpha)\right| d w \leqq B(\alpha)
$$

and

$$
\left\|T_{\alpha}^{n}\right\| \leqq 1+B(\alpha)
$$

in both spaces. Formulas (6.1), (6.3), and (6.4) show that Lemma 1 applies to the singular integral in (5.3). Hence for every $f(t) \in C_{0}[0,1]$ the sequence $T_{\alpha}^{n} f$ will converge strongly to zero. Since (6.5) holds in both spaces, we conclude that $T_{\alpha}^{n} f$ also converges strongly in $L_{1}(0,1)$ for every $f(t) \in L_{1}(0,1)\left({ }^{18}\right)$.

In order to settle the question of pointwise convergence in $L_{1}(0,1)$ we note that a dominant $G_{n}(w, \alpha)$ of $\left|P_{n}(w, \alpha)\right|$, having the properties required in Lemma 2, can be obtained from (6.3) by the simple device of increasing the right-hand sides by multiplication by suitable constants, depending upon $\alpha$ but not upon $n$ or $w$, so that the resulting estimates agree for $w=n^{-1 / \alpha}$. The new right-hand sides then form a suitable dominant having all the required properties. This completes the argument when $0<\alpha<1$.

When $\alpha=1$ several formulas from the theory of Laguerre polynomials are needed. We have first (see G. Szegö $[14$, p. 193, formulas (8.22.4) and (8.22.5)]) for $\beta>-1$

$$
\left|L_{n}^{(\beta)}(w)\right| \leqq \begin{cases}C_{1}(\beta) n^{\beta}, & \text { if } 0<n w \leqq 1, \\ C_{2}(\beta) n^{\beta / 2-1 / 4} w^{-\beta / 2-1 / 4}, & \text { if } 1<n w<n .\end{cases}
$$

These estimates give

$$
\int_{0}^{1}\left|L_{n}^{(\beta)}(w)\right| d w \leqq C(\beta) \begin{cases}n^{\beta / 2-1 / 4}, & -1<\beta<\frac{3}{2}, \\ n^{1 / 2} \log n, & \beta=\frac{3}{2}, \\ n^{\beta-1}, & \frac{3}{2}<\beta,\end{cases}
$$

and here also the reversed inequalities are valid for a suitable positive $C(\beta)$, since the inequalities in (6.6) may be reversed on subsets of measure exceeding $1 /(2 n)$ in the first case and $1 / 2$ in the second.

(18) Uniform convergence is excluded by the argument given in footnote 16 . The same remark applies to formula (6.14) below. 
Next we note that (see $[14$, p. $97,(5.1 .7)$ and $(5.1 .14)]$ )

$$
L_{n}^{(\beta)}(0)=\left(\begin{array}{c}
n+\beta \\
n
\end{array}\right)=C_{n}^{(\beta)}, \quad \frac{d}{d w} L_{n-1}^{(\beta-1)}(w)=-L_{n}^{(\beta)}(w)
$$

whence

$$
\left[L_{n}^{(\beta-1)}(0)\right]^{-1} \int_{0}^{t} L_{n}^{(\beta)}(w) d w=1-\left[L_{n-1}^{(\beta-1)}(0)\right]^{-1} L_{n-1}^{(\beta-1)}(t) .
$$

Combining (6.6) and (6.8) we see that for $\beta>\frac{1}{2}$

$$
\left[L_{n}^{(\beta-1)}(0)\right]^{-1} \int_{0}^{t} L_{n}^{(\beta)}(w) d w \rightarrow 1
$$

uniformly for $0<\epsilon \leqq t \leqq 1$.

Finally we shall need the formula

$$
L_{n}^{(\beta+k)}(w)=\sum_{\nu=0}^{n} C_{n \rightarrow \nu}^{(k-1)} L_{\nu}^{(\beta)}(w)
$$

which is an immediate consequence of the structure of the generating function of the Laguerre polynomials (see [14, formula (5.1.9)]).

Combining formula (5.3) for $\alpha=1$ with (6.7) for $\beta=1$ we see that

$$
\left\|T_{1}^{n}\right\|=O\left(n^{1 / 4}\right)
$$

and it is not difficult to show that this estimate cannot be improved. Hence strong convergence of $T_{\alpha}^{n} f$ when $n \rightarrow \infty$ is out of the question for $\alpha=1$. Let us consider $(C, k)$ summability instead. We form

$$
\sigma_{n}^{(k)}[f]=\left[C_{n}^{(k)}\right]^{-1} \sum_{\nu=0}^{n} C_{n-\nu}^{(k-1)} T_{1}^{\prime}[f] .
$$

We recall that

$$
T_{1}^{\nu}[f]=f(t)-\int_{0}^{i} L_{v-1}^{(1)}(t-u) f(u) d u .
$$

Substituting (6.13) in (6.12) and reducing with the aid of (6.10), where we take $\beta=1$ and replace $n$ by $n-1$, we obtain finally

$$
\sigma_{n}^{(k)}[f]=f(t)-\left[C_{n}^{(k)}\right]^{-1} \int_{0}^{t} L_{n-1}^{(k+1)}(t-u) f(u) d u .
$$

Formula (6.7) then shows that the norm of this transformation is bounded if and only if $k>\frac{1}{2}$. For such values of $k$ the kernel $\left[C_{n}^{(k)}\right]^{-1} L_{n-1}^{(k+1)}(w)$ then satisfies condition (ii) of Lemma 1. But condition (i) is also satisfied by virtue 
of $(6.6),(6.8)$, and $(6.9)\left({ }^{19}\right)$. Hence $\sigma_{n}^{(k)}[f]$ converges strongly to zero for every $f(t) \in C_{0}[0,1]$ when $k>\frac{1}{2}$ but not when $k \leqq \frac{1}{2}$. The extension to strong convergence in $L_{1}(0,1)$ is made as above. Pointwise convergence is settled by observing that formula (6.6) gives an admissible dominant of the kernel by increasing the factors $C_{1}(\beta)$ and $C_{2}(\beta)$ so that the two resulting estimates agree for $w=1 / n$. This completes the argument when $\alpha=1$.

The case $\alpha>1$ is entirely different. Here formula (7.11) shows that positive quantities $a(\alpha)$ and $b(\alpha)$ can be found such that in a subset of $[0,1]$ of measure greater than $1 / 2$

$$
\left|P_{n}(w, \alpha)\right|>a(\alpha) n^{1 / 2(\alpha+1)} w^{-(\alpha+2) / 2(\alpha+1)} \exp \left[b(\alpha) n^{1 /(\alpha+1)} w^{\alpha /(\alpha+1)}\right]
$$

whence follows

$$
\int_{0}^{1}\left|P_{n}(w, \alpha)\right| d w>a(\alpha) \exp \left[\frac{1}{2} b(\alpha) n^{1 /(\alpha+1)}\right]
$$

for all large $n$. This implies that $\left\|T_{\alpha}^{n}\right\|$ is of the same order of magnitude for large $n$, that is, condition (3.11) of Theorem 7 , which is necessary for $(C, k)$ summability, is violated for all $k$ as soon as $\alpha>1$. We know already from Theorem 10 that the other necessary condition (3.10) is violated for $\alpha \geqq 2$. Thus the sequence $T_{\alpha}^{n}$ is not $(C)$-ergodic for $\alpha>1$ and the proof of Theorem 11 is complete.

7. Asymptotic behavior of $P_{n}(w, \alpha)$ and related functions $\left({ }^{20}\right)$. By definition

$$
P_{n}(w, \alpha)=\sum_{\nu=1}^{n}(-1)^{\nu-1}\left(\begin{array}{l}
n \\
\nu
\end{array}\right) \frac{w^{\nu \alpha-1}}{\Gamma(\nu \alpha)} .
$$

We introduce the entire function

$$
H_{\alpha}(z)=\sum_{\nu=1}^{\infty} \frac{(-1)^{\nu-1}}{\nu ! \Gamma(\nu \alpha)} z^{\nu}
$$

which is an obvious generalization of the Bessel function $J_{1}$. Indeed

$$
H_{1}(z)=z^{1 / 2} J_{1}\left(2 z^{1 / 2}\right) \text {. }
$$

A simple argument shows that if $n \rightarrow \infty$ and $w \rightarrow 0$ in such a manner that $w^{\alpha} n \rightarrow z \geqq 0$ then

$$
w P_{n}(w, \alpha) \rightarrow H_{\alpha}(z), \quad w^{\alpha} n \rightarrow z .
$$

Hence for $w^{\alpha} n=O(1)$ we have $w P_{n}(w, \alpha)=O\left(w^{\alpha} n\right)$ or

$$
\left|P_{n}(w, \alpha)\right| \leqq A(\alpha) w^{\alpha-1} n \text { when } 0<w^{\alpha} n \leqq 1 .
$$

(19) In formula (6.9) we may obviously replace one of the subscripts $n$ by $n-1$ without affecting the conclusion.

(20) This section of the paper is entirely due to Professor G. Szegö, who has also contributed to the results of $\S 6$. 
This is the first of the inequalities in (6.3) above. The second inequality requires a rather elaborate argument.

We assume next $w=O(1)$ and put

$$
\eta=\left(\frac{w}{n}\right)^{\alpha /(\alpha+1)}
$$

with the understanding that $\eta n \rightarrow \infty$. Using Hankel's integral for the Gamma function, we get after some simplifications

$$
P_{n}(w, \alpha)=-\frac{1}{2 \pi i w} \int_{-\infty}^{(0+)} e^{t}\left\{1-\left(\frac{w}{t}\right)^{\alpha}\right\}^{n} d t .
$$

Here the path of integration is a loop surrounding the negative real axis and $t=0$ counterclockwise and $t^{\alpha}$ is real positive when $t$ is so. Substitution of $t=\eta n \tau$ gives

$$
P_{n}(w, \alpha)=-\frac{\eta n}{2 \pi i w} \int_{-\infty}^{(0+)}\left[e^{n \tau}\left(1-\eta \tau^{-\alpha}\right)\right]^{n} d \tau
$$

Putting

$$
f_{\eta}(\tau)=\eta \tau+\log \left(1-\eta \tau^{-\alpha}\right)
$$

we have

$$
\begin{aligned}
& f_{\eta}^{\prime}(\tau)=\eta+\frac{\eta \alpha \tau^{-\alpha-1}}{1-\eta \tau^{-\alpha}}, \\
& f_{\eta}^{\prime \prime}(\tau)=-\eta \alpha \frac{(\alpha+1) \tau^{-\alpha-2}\left(1-\eta \tau^{-\alpha}\right)+\eta \alpha \tau^{-2 \alpha-2}}{\left(1-\eta \tau^{-\alpha}\right)^{2}},
\end{aligned}
$$

where the primes denote differentiation with respect to $\tau$.

Let $S$ be the root of the equation $f_{\eta}^{\prime}(\tau)=0$ which tends to

$$
S_{0}=\alpha^{1 /(\alpha+1)} e^{\pi i /(\alpha+1)}
$$

when $\eta \rightarrow 0$. This root can be expanded in powers of $\eta$ for small $\eta$,

$$
S=S_{0}+S_{1} \eta+S_{2} \eta^{2}+\cdots \text {. }
$$

We have then

$$
\begin{aligned}
f_{\eta}^{\prime \prime}(S) & =\eta\left[(\alpha+1) S^{-1}-\eta\right]=(\alpha+1) \alpha^{-1 /(\alpha+1)} e^{-\pi i /(\alpha+1)} \eta+O\left(\eta^{2}\right), \\
f_{\eta}(\tau) & =f_{\eta}(S)+\frac{1}{2} \eta(\tau-S)^{2}\left[(\alpha+1) S^{-1}+\Delta(\tau, \eta)\right],
\end{aligned}
$$

where $\Delta(\tau, \eta)$ is holomorphic in a neighborhood of $\tau=S$ and $|\Delta|$ is uniformly small provided $\eta$ and $\max |\tau-S|=M$ are sufficiently small, $M$ being independent of $\eta$.

Let $0<\alpha<1$. Then $\pi / 2<\pi /(\alpha+1)<\pi$. We deform the loop into a contour consisting of the two rays arg $\tau= \pm \arg S,|\tau| \geqq \delta$, plus the major arc of the 
circle $|\tau|=\delta$ joining the end points of the rays. We have then along the ray $\arg \tau=\arg S$

$$
\tau-S=(\eta n)^{-1 / 2}(\alpha+1)^{-1 / 2} i S^{1 / 2} \sigma,
$$

where

$$
\arg \sigma=\arg \left( \pm i S^{1 / 2}\right)=\frac{\pi}{2(\alpha+1)} \pm \frac{\pi}{2}+\eta^{\prime}
$$

and $\eta^{\prime} \rightarrow 0$ when $\eta \rightarrow 0$.

The customary argument in the method of steepest descents shows that the main contribution to the value of $P_{n}(w, \alpha)$ comes from the neighborhoods of $\tau=S$ and $\tau=\bar{S}$ in the integral (7.7) after the path has been deformed as indicated above and $\delta$ is chosen sufficiently small. Hence, neglecting terms of lower order, $P_{n}(w, \alpha)$ is found to be twice the real part of

$$
\begin{aligned}
-\frac{\eta n}{2 \pi i w}(\eta n)^{-1 / 2}(\alpha+1)^{-1 / 2} i S^{1 / 2}\left[\eta^{\eta S}\left(1-\eta S^{-\alpha}\right)\right]^{n} \\
\cdot \int \exp \left[-\frac{1}{2} \sigma^{2}\left(1+\Delta^{\prime}\right)\right] d \sigma .
\end{aligned}
$$

Here $\Delta^{\prime}$ is a quantity of the same character as $\Delta$. The integration with respect to $\sigma$ can be taken along the real axis and the integral tends to $(2 \pi)^{1 / 2}$ when $\eta n \rightarrow \infty$.

Expanding into powers of $\eta$ we get

$$
f_{\eta}(S) \equiv \eta S+\log \left(1-\eta S^{-\alpha}\right)=\sum_{1}^{\infty} V_{m} \eta^{m},
$$

where the $V_{m}$ depend only upon $\alpha$ and $m$. In particular,

$$
V_{1}=S_{0}-S_{0}^{-\alpha}=\left(1+\frac{1}{\alpha}\right) \alpha^{1 /(\alpha+1)} e^{\pi i /(\alpha+1)} .
$$

The series is convergent for small values of $\eta$. We note that $n \eta^{m} \rightarrow 0$ when $n \rightarrow \infty$ as soon as $m>k$ where

Hence

$$
k=\left[\frac{\alpha+1}{\alpha}\right] .
$$

$$
\left[e^{n S}\left(1-\eta S^{-\alpha}\right)\right]^{n}=\exp \left\{n \sum_{1}^{k} V_{m} \eta^{m}\right\} \cdot\left(1+\delta_{n}\right)
$$

where $\delta_{n} \rightarrow 0$ when $n \rightarrow \infty$. The first term in the finite sum gives the main contribution to the value.

In replacing $P_{n}(w, \alpha)$ by the contributions to the integral from the neighborhoods of $\tau=S$ and $\bar{S}$ we have neglected the distant parts of the path of 
integration and the neighborhood of $\tau=0$. Suppose that $\tau=\rho \exp \pi i /(\alpha+1)$ where $\rho \geqq(1+\epsilon)\left|S_{0}\right|, \epsilon$ a fixed positive number independent of $n$. The contribution of a circular arc connecting $(1+\epsilon) S$ and $(1+\epsilon) S_{0}$ may be neglected. We can then replace $\log \left(1-\eta \tau^{-\alpha}\right)$ by $-\eta \tau^{-\alpha}$ with sufficient degree of accuracy and $\Re\left[f_{\eta}(\tau)\right]$ by $\eta \Re\left[\tau-\tau^{-\alpha}\right]=\eta C\left[\rho+\rho^{-\alpha}\right], C=\cos (\pi /(\alpha+1))<0$. The minimum of $\rho+\rho^{-\alpha}$ is reached for $\rho=\alpha^{1 /(\alpha+1)}=\left|S_{0}\right|=|S|+O(\eta)$. Using these observations it is found that the corresponding part of the integral in (7.7)

$$
\left|\int_{(1+\odot) S_{0}}^{\infty}\right| \leqq \exp \left\{\eta n\left[\Re\left(V_{1}\right)-\epsilon^{\prime}\right]\right\}
$$

with a fixed positive $\epsilon^{\prime}$ independent of $n$. Now let $\tau=\delta e^{i \phi}, 0 \leqq \phi \leqq \pi /(\alpha+1)$, where both $\delta$ and $\eta \delta^{-\alpha}$ are small. We can replace $\Re\left[f_{\eta}(\tau)\right]$ by $\eta \Re\left[\tau-\tau^{-\alpha}\right]$ $=\eta\left(\delta \cos \phi-\delta^{-\alpha} \cos \alpha \phi\right)$. Here the last parenthesis is an increasing function of $\phi$ which is negative for $\phi=\pi /(\alpha+1)$ and numerically as large as we please if $\delta$ has been chosen properly. The contribution from the small circle is consequently negligible in comparison with the main term. This completes the argument for $0<\alpha<1$.

The case $\alpha=1$ is already in the literature and need not be considered here. The method used above also applies to the case $1<\alpha$. Here, however, $\pi /(\alpha+1)<\pi / 2$ and we deform the loop in (7.7) into a contour consisting of the circle $|\tau|=|S|$ plus the negative real axis from $-\infty$ to $-|S|$ and back again. The main contribution still comes from the neighborhoods of $\tau=S$ and $\bar{S}$ and is given by formulas (7.9) and (7.10), where, however, $k$ is now equal to one. The discussion of the neglected parts of the path follows similar lines as above. Combining the various estimates we obtain the following result.

TheOREM 12. For $n^{-1 / \alpha}<w<\omega, 0<\alpha$,

$$
P_{n}(w, \alpha)=-\left(\frac{2}{\pi}\right)^{1 / 2}(\alpha+1)^{-1 / 2} \alpha^{1 / 2(\alpha+1)} w^{-(\alpha+2) / 2(\alpha+1)} n^{1 / 2(\alpha+1)}
$$

where

$$
\times \Re\left\{\left(1+\delta_{n}\right) \exp \left[\frac{\pi i}{2(\alpha+1)}+n\left(V_{1} \eta+\cdots+V_{k} \eta^{k}\right)\right]\right\} \text {, }
$$

$$
\eta=\left(\frac{w}{n}\right)^{\alpha /(\alpha+1)}, \quad V_{1}=\left(1+\frac{1}{\alpha}\right) \alpha^{1 /(\alpha+1)} e^{i \pi /(\alpha+1)}, \quad k=\left[1+\frac{1}{\alpha}\right],
$$

$V_{1}, \cdots, V_{n}$ depend on $\alpha$ only, and $\delta_{n} \rightarrow 0$ when $n \rightarrow \infty$.

The difference between the three cases $\alpha<1, \alpha=1, \alpha>1$ is rather striking. For $\alpha<1$ the exponential factor gives rise to oscillations with a strong damping factor. For $\alpha=1$, we have $k=2$ and $n V_{1} \eta=2(w n)^{1 / 2} i, n V_{2} \eta^{2}=\frac{1}{2} w$, so that the change in amplitude depends upon $w$ but not upon $n$, except for the trivial factor $n^{1 / 4}$. For $\alpha>1$ the exponential factor is still oscillatory but now the 
amplitudes tend very rapidly to infinity with $n$. Formulas (6.3), second line, and (6.15) are immediate consequences of (7.11).

The same method can be applied to the function $H_{\alpha}(z)$ of $(7.2)$ and leads to the estimate

$$
H_{\alpha}(z)=-\left(\frac{2}{\pi}\right)^{1 / 2}(\alpha+1)^{-1 / 2}(\alpha z)^{1 / 2(\alpha+1)}
$$

$$
\times \Re\left\{[1+\delta(z)] \exp \left[\frac{\pi i}{2(\alpha+1)}+\left(1+\frac{1}{\alpha}\right)(\alpha z)^{1 /(\alpha+1)} e^{\pi i /(\alpha+1)}\right]\right\}
$$

where $\delta(z) \rightarrow 0$ when $z \rightarrow+\infty$. In particular, if $0<\alpha<1$,

$$
\left|H_{\alpha}(z)\right|<\exp \left[-c z^{1 /(\alpha+1)}\right], \quad c>0, \quad z \geqq 1 .
$$

For $\alpha>0, H_{\alpha}(z)$ has only real non-negative zeros and the same is true in the case of the polynomials

$$
\Phi_{n}(z, \alpha)=\sum_{\nu=1}^{n}(-1)^{\nu-1}\left(\begin{array}{l}
n \\
\nu
\end{array}\right) \frac{z^{\nu}}{\Gamma(\nu \alpha)} .
$$

(See G. Pólya and G. Szegö [12, vol. 2, p. 68, problem 167].) We note that

$$
P_{n}(w, \alpha)=w^{-1} \Phi_{n}\left(w^{\alpha}, \alpha\right)
$$

and

$$
\Phi_{n}(z, 1)=z L_{n-1}^{(1)}(z)
$$

\section{BiBLIOGRAPHY}

1. A. F. Andersen, Studier over Cesdro's Summabilitetsmetode, Thesis, Copenhagen, 1921, 100 pp.

2. S. Banach, Theorie des operations lineaires, Warsaw, 1932.

3. G. D. Birkhoff, Proof of the ergodic theorem, Proc. Nat. Acad. Sci. U.S.A. vol. 17 (1931) pp. $656-660$.

4. S. Bochner, Integration von Funktionen, deren Werte die Elemente eines Vektorraumes sind, Fund. Math. vol. 20 (1933) pp. 262-276.

5. S. Chapman, On non-integral orders of summability of series and integrals, Proc. London Math. Soc. (2) vol. 9 (1911) pp. 369-409.

6. L. W. Cohen, On the mean ergodic theorem, Ann. of Math. (2) vol. 41 (1940) pp. 505-509.

7. N. Dunford, Spectral theory. I. Convergence to projections, Trans. Amer. Math. Soc. vol. 54 (1943) pp. 185-217.

7a. - Spectral theory, Bull. Amer. Math. Soc. vol. 49 (1943) pp. 637-651.

8. E. Hille, Notes on linear transformations. II. Analyticity of semi-groups, Ann. of Math. (2) vol. 40 (1939) pp. 1-47.

9. E. Hille and J. D. Tamarkin, On the theory of linear integral equations. I. Ann. of Math. (2) vol. 31 (1930) pp. 479-528.

10. J. E. Littlewood, The converse of Abel's theorem on power series, Proc. London Math. Soc. (2) vol. 9 (1911) pp. 434-448. 
11. J. von Neumann, Proof of the quasi-ergodic hypothesis, Proc. Nat. Acad. Sci. U.S.A. vol. 18 (1932) pp. 70-82.

12. G. Polya and G. Szegö. Aufgaben und Lehrsätze aus der Analysis, 2 vols., Berlin, 1925.

13. M. Riesz, Über einen Satz des Herrn Fatou, Journal für Mathematik vol. 140 (1911) pp. 89-99.

14. G. Szegö, Orthogonal polynomials, Amer. Math. Soc. Colloquium Publications, vol. 23, New York, 1939.

15. N. Wiener, Tauberian theorems, Ann. of Math. (2) vol. 33 (1932) pp. 1-100.

16. A. Wiman, Über die Nullstellen der Funktionen $E_{\alpha}(x)$, Acta Math. vol. 29 (1905) pp. 217-234.

YALE UNIVERSITY, New Haven, Conn. 